ORIGINAL ARTICLE

\title{
Immunohistochemical prognostic index for breast cancer in young women
}

\author{
I Guerra, J Algorta, R Díaz de Otazu, A Pelayo, J Fariña
}

See end of article for authors' affiliations

......................

Correspondence to: Dr I Guerra, Servicio Anatomía Patológica, Hospital Txagorritxu, c/ José Achótegui $s / \mathrm{n}$, 01009 Vitoria-Gasteiz, Spain; iguerra@htxa. osakidetza.net

Accepted for publication 4 August 2003

\begin{abstract}
Aims: Women under 35 years of age comprise a small proportion of patients with breast cancer, but determining their prognosis can be difficult. This prospective, multivariate study looked at several factors with the aim of obtaining a useful index to evaluate the prognosis of these women.

Methods: In total, 108 patients below 35 years of age affected by invasive ductal carcinoma without distant metastasis were studied. The mean duration of the follow up period was six years. Histopathological (tumour size, histological grade, and lymph node stage) and immunohistochemical (c-erbB-2, p53, oestrogen receptor, and progesterone receptor) factors were measured in all patients, and the Nottingham prognostic index (NPI) was then calculated. An immunohistochemical prognostic index (IHPI) was created using the arithmetic sum of the four individual immunohistochemical factors.

Results: In univariate assessment of survival, all the studied factors yielded a significant association with either overall survival or disease free survival, except for c-erbB-2 and p53 with disease free survival. In univariate calculation of risk, all the factors gave significant results; however, in multivariate analysis only tumour size, histological grade, and progesterone receptor were significant. Both NPI and IHPI correlated significantly with prognosis. In multivariate regression analysis, IHPI correlated with tumour size and there was a significant interaction between both variables.

Conclusion: IHPI is very useful in determining the prognosis of tumours $\leqslant 2 \mathrm{~cm}$ and of moderate use for tumours $>2$, although it has no use in tumours $>5 \mathrm{~cm}$.
\end{abstract}

W omen under 35 years of age form only a small proportion of patients suffering from breast cancer $(5 \%),{ }^{1}$ but their prognosis can be complicated because of delayed diagnosis resulting from the unexpectedness and severity of the disease.

An accurate prognosis is necessary not only to determine the natural history of the disease but also to establish the appropriate treatment. A better prognostic stratification of patients is important so that patients can receive treatment at an earlier stage of the diagnosis and to avoid unnecessary risk to those patients who do not need additional treatment.

Several prognostic factors that provide information about either survival or risk of recurrence are available for breast cancer, namely: tumour size, axillary lymph nodes affected, histological type and grade, and hormone receptor analysis. ${ }^{2}$ Furthermore, prognostic indexes, which combine several factors, have been devised and have been available for many years. The most widely applied index, the Nottingham prognosis index (NPI) was published in $1982^{3}$ and revised 10 years later. ${ }^{4}$ Recently, as a result of the development of immunohistochemical techniques to measure oncogene expression, ${ }^{56}$ new markers of breast cancer have been used, alone or in combination with other prognostic factors.

\footnotetext{
"A better prognostic stratification of patients is important so that patients can receive treatment at an earlier stage of the diagnosis and to avoid unnecessary risk to those patients who do not need additional treatment"
}

We report the results of a prospective, multivariate study of several immunohistochemical factors with the aim of obtaining a useful index to evaluate the prognosis of young women affected by breast cancer.

\section{MATERIAL AND METHODS \\ Patients}

In total, 108 patients below 35 years of age (mean age, 32; range, 18-35) affected by invasive ductal carcinoma without distant metastasis were studied. The mean duration of follow up was six years (range, 1-18 years).

Clinicoepidemiological data were obtained from the patients' records. Thirteen of the patients (12\%) had antecedents of breast cancer in first degree relatives and another 13 in second degree relatives. In addition, another 21 patients had relatives suffering from other neoplastic diseases. Seventy four of the patients $(68 \%)$ had children, and 55 had breast fed them. Nine patients (6\%) were pregnant or breast feeding at the time of diagnosis. In 18 patients $(17 \%)$ the tumour was bilateral. Surgery was performed whenever possible as conservative tumorectomy (29 patients), radical mastectomy (63 patients), or postchemotherapy (12 patients). Additional chemotherapy was administered in 71 patients.

\section{Histopathological study}

Tumour size was measured in the surgical specimens before the preparation of histological sections, except in the case of locally advanced carcinoma, in which size was measured by imaging techniques (mammography and ultrasound before chemotherapy). Tumours were subsequently classified into three groups according to size $(\leqslant 2 \mathrm{~cm} ;>2-5 \mathrm{~cm} ;>5 \mathrm{~cm})$.

Specimens were also classified according to histological grade $^{7}$ into grade I (well differentiated), grade II (moderately differentiated), or grade III (poorly differentiated).

Abbreviations: ER, oestrogen receptor; IHPI, immunohistochemical prognostic index; NPI, Nottingham prognostic index; PR, progesterone receptor 
Lymph node stage was also classified into grade 1 (not affected), grade 2 (one to three affected nodes), or grade 3 (four or more affected nodes). Patients suffering from locally advanced carcinoma were considered as a differentiated group.

\section{Immunohistochemical study}

Immunohistochemistry was performed on representative paraffin wax embedded specimens from the main tumour. In brief, $5 \mu \mathrm{m}$ thick histological sections were mounted on poly-L-lysine treated slides, maintained at $60^{\circ} \mathrm{C}$ for 18 hours, and subsequently dewaxed and rehydrated. Non-specific binding sites were blocked with immersion in a solution of $3 \% \mathrm{H}_{2} \mathrm{O}_{2}$ in methanol for 10 minutes. For antigen retrieval, slides were transferred to citrate buffer and boiled for 10 minutes in a microwave oven.

Tumour specimens were analysed using the following antibodies: anti-c-erbB-2 (Dako, Glostrup, Denmark; clone CB1 1; 1/200 dilution for one hour at room temperature), antip53 (NovoCastra, Newcastle, UK; clone DO7; 1/50 dilution for one hour at room temperature), anti-oestrogen receptor (ER) (Zymed Laboratories, South San Francisco, California, USA; prediluted monoclonal mouse anti-oestrogen receptor clone 1D5, incubated for 18 hours in a moist chamber at $4^{\circ} \mathrm{C}$ ), and anti-progesterone receptor (PR) (Zymed Laboratories; clone $1 \mathrm{~A} 6,1 / 40$ dilution for 18 hours in a moist chamber at $4^{\circ} \mathrm{C}$ ). In addition, positive and negative controls for each marker were included.

Sections were then washed in phosphate buffered saline, stained with streptavidin-biotin-peroxidase complex, and then incubated for 20 minutes at room temperature. The stain was developed using aminoethylcarbazol. Interpretation of the staining was carried out according to the usual criteria by the same experienced pathologist. In brief, c-erbB-2 was scored positive if at least $50 \%$ of tumour cell membranes were stained; p53, ER, and PR were scored positive, as described previously, ${ }^{8}$ if more than $10 \%$ of tumour cell nuclei were immunoreactive, in the evaluation of 10 random microscopic fields comprising 200 tumorous cells.

\section{Prognostic indexes}

The NPI was calculated for all cases using the following formula: NPI $=0.2 \times$ tumour size $(\mathrm{cm})+$ histological grade (1-3: good, moderate, poor) + lymph node stage (1-3 by grade); the higher the index the worse the prognosis. ${ }^{4}$ Patients with locally advanced carcinoma were excluded from the calculation. The patients were grouped into three prognostic groups according to the NPI results.

The proposed immunohistochemical prognostic index (IHPI) was constructed as follows: one point was assigned for each positive value for either c-erbB-2 or p53 and zero points were given for negative values; one point was given for any negative value of ER or PR and zero points were given when positive. Tumours were then ranked from 0 to 4 points (the higher the index, the worse the prognosis), and grouped into good prognosis (0-1 points), moderate prognosis (2 points), or poor prognosis (3-4 points).

\section{Statistical methods}

Associations between pairs of individual markers were calculated by the non-parametric Kendall's $\tau$-b correlation test. For each factor studied, univariate Kaplan-Meier analysis for both relapse free and overall survival was performed and comparisons of distributions were performed with the log rank test.

Therefore, variables were first included in a univariate and two multivariate Cox regression models (one including the three histopathological factors and the other including the four immunohistochemical factors) to analyse which factors were associated with survival and relapse free period to compute the relative risk of mortality rate and disease progression rate. For that analysis, the value of the continuous variable tumour size (not categorised) was included in the calculation. In addition, the relation between the proposed IHPI index and histopathological factors was also analysed by another Cox regression model.

Significance was set at $\mathrm{p}<0.05$.

\section{RESULTS}

Median (SEM) overall survival at two, five, and 10 years were 84.5 (3.6), 54.7 (5.4), and 38.7 (5.8) months, respectively. Median (SEM) disease free periods at two, five, and 10 years were 64.4 (4.7), 44.2 (5.1), and 38.2 (5.3) months, respectively. None of the clinical or epidemiological factors studied (familial history, age, parity, or lactation) influenced prognosis. When patients were classified by the NPI, nine had a good prognosis, 45 a moderate prognosis, and 28 a poor prognosis. The remaining 26 patients were affected by locally advanced carcinoma. With regard to IHPI, 19 patients scored no points, 13 scored one point, 23 patients two points, 31 cases three points, and 22 patients scored four points.

The relation between pairs of the analysed histopathological and immunohistochemical factors was analysed, and an association was found between all the pairs of factors analysed except for p53 and PR (tables 1 and 2).

With regard to the survival assessment, on univariate analysis all the histopathological factors (table 3 ) and all the immunohistochemical factors (table 4 ) yielded a significant association with either overall survival or disease free survival, except c-erbB-2 and p53 with disease free survival. When combining the histopathological factors (NPI) or immunohistochemical markers (IHPI), as described earlier, there was also a clear and significant association between both indexes and survival. Figure 1 shows actuarial curves for both indexes. In addition, the risk associated with each factor and prognostic index was calculated as odds ratios by both univariate and multivariate analysis (tables 5 and 6).

Multivariate regression analysis was subsequently applied to see whether the IHPI correlated with the histopathological factors. The best association was found with tumour size and this model was significantly associated with prognosis ( $p<0.0001$ for both overall and disease free survival). There was a significant interaction between both variables, reflecting the fact that IHPI is very useful in the prognosis of small tumours $\leqslant 2 \mathrm{~cm}$ in size (overall survival, $\mathrm{OR}=3.0$;

Table 1 Correlation between histopathological factors

\begin{tabular}{lcl}
\hline Histopathological factors & Histological grade & Lymph node stage \\
\hline Tumour size & $0.26(p<0.01)$ & $0.58(p<0.001)$ \\
Histological grade & & $0.30(p<0.001)$
\end{tabular}

The values shown are Kendall's $\tau$-b coefficient and $p$ value.

Table 2 Correlation between immunohistochemical factors

\begin{tabular}{llll}
\hline $\begin{array}{l}\text { Immunohistochemical } \\
\text { factors }\end{array}$ & $\mathrm{p53}$ & $\mathrm{ER}$ & $\mathrm{PR}$ \\
\hline c-erbB-2 & 0.39 & 0.34 & 0.43 \\
& $(p<0.001)$ & $(p<0.001)$ & $(p<0.001)$ \\
p53 & & 0.30 & 0.13 (NS) \\
ER & & $(p<0.001)$ & 0.56 \\
& & & $(p<0.001)$ \\
\hline
\end{tabular}

The values are shown as Kendall's $\tau$-b coefficient and $p$ value. $E R$, oestrogen receptor; NS, not significant; PR, progesterone receptor. 
Table 3 Influence of histopathological markers, individually and in combination (NPI), on overall survival and disease free survival

\begin{tabular}{llll}
\hline Variable & OS & DFS & N \\
\hline $\begin{array}{lll}\text { Tumour size } \\
\leqslant 2 \mathrm{~cm}\end{array}$ & $>228$ & $>228$ & 33 \\
$>2 \leqslant 5 \mathrm{~cm}$ & $96(35)$ & $41(36)$ & 49 \\
$>5 \mathrm{~cm}$ & $23(6)$ & $12(2)$ & 26 \\
$\begin{array}{c}\text { p Value } \\
\text { Histological grade }\end{array}$ & $<0.0001$ & $<0.0001$ & \\
I/II & $>228$ & $>228$ & 52 \\
III & $57(11)$ & $29(7)$ & 56 \\
p Value & $<0.001$ & $<0.001$ & \\
Lymph node stage & & & \\
0 & $>212$ & $>212$ & 33 \\
1-3 & $100(20)$ & $71(42)$ & 26 \\
4 & $91(13)$ & $31(19)$ & 23 \\
LAC & $30(9)$ & $17(4)$ & 26 \\
p Value & $<0.0001$ & $<0.0001$ & \\
NPI & & & \\
Good & $>228$ & $>228$ & 9 \\
Moderate & $>228$ & $>228$ & 45 \\
Poor & $91(39)$ & $23(7)$ & 28 \\
LAC & $30(9)$ & $17(4)$ & 26 \\
p Value & $<0.05$ & $<0.0001$ & \\
\hline
\end{tabular}

Survival is in months (median (SEM)).

LAC, locally advanced carcinoma; NPI, Nottingham prognostic index.

disease free survival, $\mathrm{OR}=2.2$ ) and of moderate use in medium sized $(2-5 \mathrm{~cm})$ tumours (overall survival, $\mathrm{OR}=1.9$; disease free survival, $\mathrm{OR}=1.3$ ). However, IHPI could not predict the prognosis of tumours larger than $5 \mathrm{~cm}$ (overall survival, $\mathrm{OR}=0.5$; disease free survival, $\mathrm{OR}=0.5$ )

\section{DISCUSSION}

The need for a prognostic marker for breast cancer in young women to help determine treatment is without question. Although the influence of age on the prognosis of breast cancer is still controversial, with conflicting reports, ${ }^{10}{ }^{11}$ recent data have shown that young age should be considered as an adverse prognostic factor. ${ }^{12}{ }^{13}$ Furthermore, there may be socioeconomic factors relating to its occurrence in very

Table 4 Influence of immunohistochemical markers, individually and in combination (IHPI), on overall survival and disease free survival

\begin{tabular}{llll}
\hline Variable & OS & DFS & N \\
\hline c-erbB-2 & $142(17)$ & $94(17)$ & 41 \\
Negative & $58(8)$ & $31(5)$ & 67 \\
Positive & $<0.05$ & NS & \\
p Value & $100(28)$ & $71(35)$ & 75 \\
p53 & $57(6)$ & $34(7)$ & 33 \\
Negative & $<0.05$ & NS & \\
Positive & & & \\
p Value & $>212$ & $>212$ & 36 \\
ER & $57(9)$ & $31(5)$ & 72 \\
Positive & $<0.001$ & $<0.01$ & \\
Negative & & & \\
P Value & $>212$ & $>212$ & 40 \\
PR & $55(4)$ & $29(5)$ & 68 \\
Positive & $<0.0001$ & $<0.0001$ & \\
Negative & $>212$ & $>212$ & 32 \\
p Value & $96(35)$ & $34(10)$ & 23 \\
IHPI & $51(8)$ & $29(6)$ & 53 \\
Good & $<0.0001$ & $<0.001$ & \\
Moderate & Poor & & \\
p Value & & & \\
\hline
\end{tabular}

Survival is in months (median (SEM)).

$E R$, oestrogen receptor; $\mathrm{HPI}$, immunohistochemical prognostic index; LAC, locally advanced carcinoma; PR, progesterone receptor. young patients. In addition, recent reports have suggested that the presentation and characteristics of breast cancer in young women (below 35 years) can be different to that seen in older patients. ${ }^{14} 15$

Ideally, a clinically useful prognostic test should be easy to perform, reproducible, and cheap, in addition to providing information that cannot be obtained more easily by other methods. Moreover, factors should be tested by assessing the association with survival outcomes, but because this is highly dependent on sample size, the relative risk (or odds ratio) should also be provided. In addition, a prognostic factor should have independent value, as assessed by multivariate analysis. Such methodology has been applied in our present study.

Several attempts have been made to define an accurate but simple prognostic index, but the availability of new markers, such as oncogene expression, means that new indexes should be devised that assess a combination of traditional and newly developed markers. Moreover, improved and more reliable techniques should be used to simplify the rapid evaluation of specimens.

Our results confirm the usefulness of a range of classic and widely used histopathological and immunohistochemical factors in this group of patients. However, their usefulness can be dramatically increased by simply summarising them in two easy to calculate indexes, the classic widely applied $\mathrm{NPI}^{11}$ and the proposed IHPI.

It is well known that the usual prognostic factors are age dependent, resulting in a worse prognosis in younger patients. Our present results agree with those obtained by other authors who have studied young women, whereas they differ from those obtained in older patients. ${ }^{16-18}$

The usefulness of the NPI has been clearly established in prospective studies of large numbers of patients, ${ }^{411} 19$ and those results are also reflected in our series. NPI has a high predictive value both for overall survival and disease free survival, which can be improved by combining the same factors in a multivariate analysis. ${ }^{20}$

It is noteworthy that the immunohistochemical factors had a high prognostic value in this group of young patients. It has been shown previously that c-erbB-2 and p53 are overexpressed more frequently in younger women, ${ }^{21} 22$ whereas ER and PR are more frequently positive in older patients. ${ }^{1}$

The combination of immunohistochemical markers in the IHPI used here was a simple and reliable way to predict prognosis in our series or patients. Furthermore, a more accurate prognosis could be obtained when calculating both complementary indexes together.

The proposed index displays the desired characteristics of a prognostic index by being simple to calculate and clinically useful, and it provides information independent of other factors, such as tumour size, which is known to have a significant impact.

\section{"The combination of immunohistochemical markers in the immunohistochemical prognostic index used here was a simple and reliable way to predict prognosis in our series or patients"}

The proposed index is based on simple, commonly performed procedures that can be carried out in most routine pathology laboratories using commercially available reagents. The immunohistochemical evaluation of hormonal receptors (ER and PR) has clear advantages over the classic binding assays, such as a lower rate of false positives and the possibility of using pretreated specimens. Furthermore, paraffin wax embedded specimens are widely available so 
A

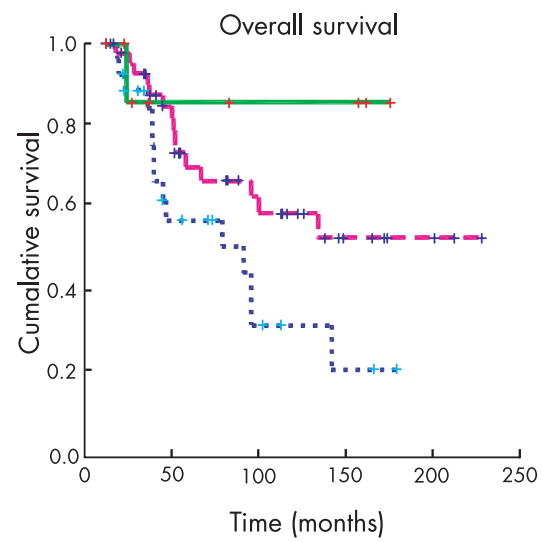

B

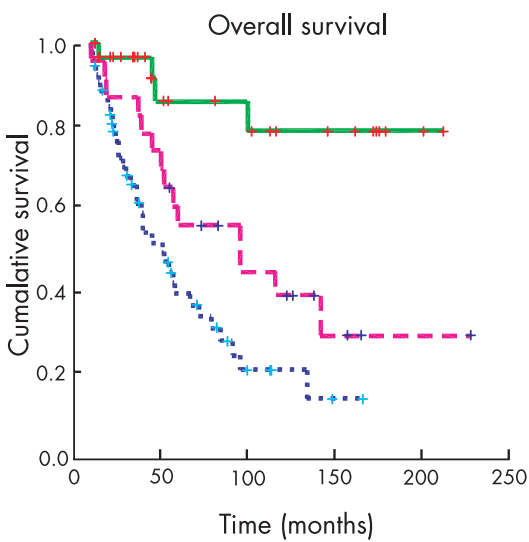

Figure 1 Actuarial curve for overall survival (left) and disease free survival (right), according to (A) Nottingham prognostic index scores or (B) immunohistochemical prognostic index scores.

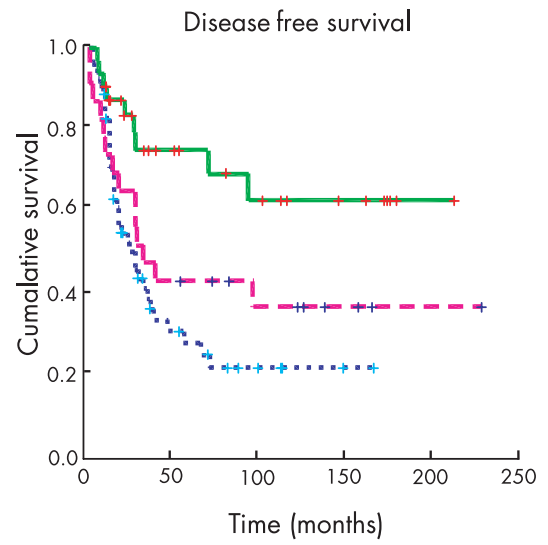

that, in the future, new immunohistochemical markers could very simply be added to the IHPI or even replace any of those currently used if the index is improved.

The IHPI was significantly related to prognosis in those tumours $\leqslant 2 \mathrm{~cm}$ in size, whereas there was only a moderate influence in the tumours with a size of $>2-5 \mathrm{~cm}$ and even a lack of association in those tumours $>5 \mathrm{~cm}$. However, in women under 35 years of age, the diagnosis is usually made on small tumours of less than $2 \mathrm{~cm}$ in diameter, where the IHPI is, in fact, more useful and provides more information. Moreover, prognostic factors are needed more for the assessment of small tumours than large tumours, where the prognosis is usually evident. In addition, the IHPI was specifically assessed for use in young women.

We used the IHPI as a complementary index to the NPI, the most widely applied prognostic index, to improve its discriminatory power to detect risk (overall and disease free survival) in the four groups of patients studied (poor, moderate, and good prognosis and locally advanced carcinoma).

To make the calculation of the IHPI easy, we simply added together the points obtained for the four individual factors (c-erbB-2, p53, ER, and PR). Hypothetically, the value of the IHPI could be increased by weighting the value of each

\begin{tabular}{|c|c|c|c|c|}
\hline \multirow[b]{2}{*}{ Factor } & \multicolumn{2}{|c|}{ Overall survival } & \multicolumn{2}{|c|}{ Disease free survival } \\
\hline & OR & Significance & OR & Significance \\
\hline Tumour size & 1.320 & +H+ & 1.249 & +H \\
\hline Histological grade & 3.011 & +++ & 2.528 & ++ \\
\hline Lymph node stage & 1.849 & +H+ & 1.677 & +H \\
\hline NPI & 1.553 & ++ & 1.529 & ++ \\
\hline c-erbB-2 & 2.185 & + & 1.606 & - \\
\hline p53 & 1.777 & + & 1.391 & - \\
\hline ER & 3.549 & ++ & 2.323 & ++ \\
\hline PR & 5.441 & +++ & 3.319 & +++ \\
\hline IHPI & 1.707 & +H+ & 1.389 & ++ \\
\hline
\end{tabular}


Table 6 Multivariate analysis of risk associated with each individual factor and prognostic index for overall survival and disease free survival

\begin{tabular}{|c|c|c|c|c|}
\hline \multirow[b]{2}{*}{ Factor } & \multicolumn{2}{|c|}{ Overall survival } & \multicolumn{2}{|c|}{ Disease free survival } \\
\hline & OR & Significance & OR & Significance \\
\hline Tumour size & 1.266 & +++ & 1.171 & ++ \\
\hline Histological grade & 2.295 & ++ & 1.940 & + \\
\hline Lymph node stage & 1.152 & - & 1.209 & - \\
\hline$c$-erbB-2 & 1.292 & - & 0.939 & - \\
\hline p53 & 1.223 & - & 1.153 & - \\
\hline ER & 1.869 & - & 1.219 & - \\
\hline PR & 3.633 & ++ & 2.991 & ++ \\
\hline
\end{tabular}

\section{Take home messages}

- We devised an immunohistochemical prognostic index (IHPI) for breast cancer in young women (less than 35 years), which measured the expression of p53, c-erbB2 , the oestrogen receptor, and the progesterone receptor

- This IHPI was very useful in determining the prognosis of tumours $\leqslant 2 \mathrm{~cm}$ and of moderate use for tumours $>2-5$, although it had no use in tumours $>5 \mathrm{~cm}$

- Used in conjunction with the Nottingham prognostic index, the IHPI can improve the discriminatory power to detect risk (overall and disease free survival) in these patients

component in the calculation, without making the calculation much more complicated. Further prospective and collaborative studies including higher numbers of patients are needed to confirm the usefulness of the IHPI and to evaluate the weight of each component.

In conclusion, the authors provide a solid rationale for clinical decisions based on a combination of common and reliable techniques that are summarised in the IHPI. The proposed IHPI can be usefully applied in clinical practice to help define prognosis in young women with breast carcinoma, particularly those with tumours of $\leqslant 2 \mathrm{~cm}$ in size.

\section{ACKNOWLEDGMENTS}

The authors thank MC Albaina for carrying out the immunohistochemical analyses, and Drs MJ Mitxelena, E Soga, and R Rezola for their contribution to the clinical evaluations. The authors are also indebted to Dr G Bautista for his continuous support.

\section{Authors' affiliations}

I Guerra, R Díaz de Otazu, Servicio de Anatomía Patológica, Hospital Txagorritxu, c/ José Achótegui s/n, 01009 Vitoria-Gasteiz, Spain J Algorta, Unidad Ensayos Clínicos (Fundación LEIA), Hospital Txagorritxu

A Pelayo, J Fariña, Cátedra de Anatomía Patológica II. Hospital Clínico de San Carlos, 28972 Madrid, Spain

\section{REFERENCES}

1 Kotari AS, Fentiman IS. Breast cancer in young women. Int J Clin Pract 2002;56: 184-7.

2 Elston CW, Ellis IO, Pinder SE. Pathological prognostic factors in breast cancer. Crit Rev Oncol Hematol 1999;31:209-23.

3 Haybittle JL, Blamey RW, Elston CW, et al. A prognostic index in primary breast cancer. Br J Cancer 1982;45:361-6.

4 Galea MH, Blamey RW, Elston CE, et al. The Nottingham prognostic index in primary breast cancer. Breast Cancer Res Treat 1992;22:207-19.

5 Siögren S, Inganäs $M$, Lindgren $L$, et al. Prognostic and predictive value of cerbB-2 overexpression in primary breast cancer, alone and in combination with other prognostic markers. J Clin Oncol 1998;16:462-9.

6 Rosen PP, Lesser ML, Arroyo CD, et al. p53 in node-negative breast carcinoma: an immunohistochemical study of epidemiologic risk factors, histologic features, and prognosis. J Clin Oncol 1995;13:821-30.

7 Elston CW, Ellis IO. Pathological prognostic factors in breast cancer. I. The value of histological grade in breast cancer: experience from a large study with long-term follow-up. Histopathology 1991;19:403-10.

8 Pinto $\mathrm{AE}$, André S, Pereira T, et al. c-erbB-2 oncoprotein overexpression identifies a subgroup of estrogen receptor positive $(E R+)$ breast cancer patients with poor prognosis. Ann Oncol 2001;12:525-33.

9 Ferrero-Pous M, Trassard M, Le Doussal V, et al. Comparison of enzyme immuno assay and immunoshistochemical measurements of estrogen and progesterone receptors in breast cancer patients. Appl Immunohistochem Mol Morphol $2001 ; 9: 267-75$.

10 Holli K, Isola J. Effect of age on the survival of breast cancer patients. Eur J Cancer 1997;33:425-8.

11 Kollias J, Elston CW, Ellis IO, et al. Early-onset breast cancerhistopathological and prognostic considerations. $\mathrm{Br} J$ Cancer 1997;75:1318-23.

12 Dubsky PC, Gnant MF, Taucher S, et al. Young age as an independent adverse prognostic factor in premenopausal patients with breast cancer. Clin Breast Cancer 2002;3:65-72.

13 Love RR, Duc NB, Dinh NV, et al. Young age as an adverse prognostic factor in premenopausal women with operable breast cancer. Clin Breast Cancer 2002;2:294-8.

14 Colleoni M, Rotmensz N, Robertson C, et al. Very young women ( $<35$ years) with operable breast cancer: features of disease at presentation. Ann Oncol 2002;13:273-9.

15 Bertheau P, Seinberg SM, Cowan K, et al. Breast cancer in young women: clinicopathologic correlation. Semin Diagn Pathol 1999;16:248-56.

16 De La Rochefordière A, Asselain B, Campana F, et al. Age as prognostic factor in premenopausal breast carcinoma. Lancet 1993;341:1039-43.

17 Fowble BL, Schultz D, Overmoyer B, et al. The influence of young age on outcome in early stage breast cancer. Int $J$ Radiat Oncol Biol Phys 1994;30:23-33

18 Kurtz JM, Spitalier JM, Amalric R, et al. Mammary recurrences in women younger than forty. Int J Radiat Oncol Biol Phys 1988;15:271-6.

19 Kollias J, Murphy CA, Elston CW, et al. The prognosis of small primary breast cancers. Eur $J$ Cancer 1999;35:908-12.

20 Anderson TJ. Breast cancer prognostication in the 21 st century and the Nottingham prognostic index. J Clin Pathol 2002;55:86-7.

21 Walker RA, Lees E, Webb MB, et al. Breast carcinomas occurring in young women $(<35)$ are different. Br J Cancer 1996;74:1796-800.

22 Bertheau P, Seinberg SM, Merino MJ. C-erbB2, p53 and nm23 gene product expression in breast cancer of young women: immunohistochemical analysis and clinicopathologic correlation. Hum Pathol 1998;29:323-9. 